\title{
Paced Ventricular Rhythm without Intrinsic Ventricular Complexes
}

National Cancer Institute

\section{Source}

National Cancer Institute. Paced Ventricular Rhythm without Intrinsic Ventricular

Complexes. NCl Thesaurus. Code C100061.

An electrocardiographic finding that the cardiac rhythm is initiated by a cardiac

pacemaker generated electrical impulse in the ventricle. Intrinsic ventricular complexes are absent. 